Research Paper

\title{
Combined B-type Natriuretic Peptide as strong predictor of short-term mortality in patients after Liver Transplantation
}

Hyun Sik Chung ${ }^{1}$, AMi Woo ${ }^{1}$, Min Suk Chae'², Sang Hyun Hong'², Chul Soo Park ${ }^{2}$, Jong Ho Choi², Yun Sung Jo ${ }^{3 凶}$

1. Department of Anesthesiology and Pain Medicine, Eunpyeong St. Mary's Hospital, College of Medicine, The Catholic University of Korea, Seoul, Republic of Korea.

2. Department of Anesthesiology and Pain Medicine, Seoul St. Mary's Hospital, College of Medicine, The Catholic University of Korea, Seoul, Republic of Korea.

3. Department of Obstetrics and Gynecology, St. Vincent's Hospital, College of Medicine, The Catholic University of Korea, Gyeonggi, Republic of Korea.

$\triangle$ Corresponding author: Yun Sung Jo, M.D., Ph.D., Department of Obstetrics and Gynecology, St. Vincent's Hospital, The Catholic University of Korea, 93 Jungbu-daero, Ji-dong, Paldal-gu, Suwon, Gyeonggi 16247, Republic of Korea; E-mail: eggs76@catholic.ac.kr, Tel: +82-31-249-7310, Fax: +82-2-2030-3861.

(C) The author(s). This is an open access article distributed under the terms of the Creative Commons Attribution License (https://creativecommons.org/licenses/by/4.0/). See http://ivyspring.com/terms for full terms and conditions.

Received: 2020.10.08; Accepted: 2021.04.14; Published: 2021.04.23

\begin{abstract}
Background: B-type natriuretic peptide (BNP) is a well-known predictor for prognosis in patients with cardiac and renal diseases. However, there is a lack of studies in patients with advanced hepatic disease, especially patients who underwent liver transplantation (LT). We evaluated whether BNP could predict the prognosis of patients who underwent LT.
\end{abstract}

Material and Methods: The data from a total of 187 patients who underwent LT were collected retrospectively. The serum levels of BNP were acquired at four time points, the pre-anhepatic ( $\mathrm{Tl} 1)$, anhepatic (T2), and neohepatic phases (T3), and on postoperative day 1 (T4). The patients were dichotomized into survival and non-survival groups for 1-month mortality after LT. Combined BNP (cBNP) was calculated based on conditional logistic regression analysis of pairwise serum BNP measurements at two time points, T2 and T4. The area under the receiver operating characteristic curve (AUROC) was analyzed to determine the diagnostic accuracy and cut-off value of the predictive models, including cBNP.

Results: Fourteen patients $(7.5 \%)$ expired within one month after LT. The leading cause of death was sepsis ( $N=9,64.3 \%)$. The MELD and MELD-Na scores had an acceptable predictive ability for 1-month mortality (AUROC $=0.714$, and 0.690 , respectively). The BNPs at each time point $(T 1-T 4)$ showed excellent predictive ability (AUROC $=0.864,0.962,0.913$, and 0.963 , respectively). The cBNP value had an outstanding predictive ability for 1 -month mortality after LT (AUROC $=0.976)$. The optimal cutoff values for cBNP at T2 and T4 were 137 and 187, respectively.

Conclusions: The cBNP model showed the improved predictive ability for mortality within 1-month of LT. It could help clinicians stratify mortality risk and be a useful biomarker in patients undergoing LT.

Key words: B-type natriuretic peptide; liver transplantation; mortality; prognosis; sensitivity; specificity

\section{Introduction}

Several established scoring systems, including a model for Child-Turcotte-Pugh (CTP), end-stage liver disease (MELD), and sequential organ failure assessment (SOFA), have been introduced to estimate the mortality in patients undergoing liver transplantation (LT) [1-3]. With respect to the MELD score, the pretransplant MELD score is also a well-known predictive model for estimating the early mortality of patients undergoing LT [4]. Other than these scoring systems, some biomarkers, such as proteinuria have been used to estimate post-LT mortality [5]. With respect to cardiac markers, previous studies have been reported a correlation between serum troponin levels and cardiovascular 
events or graft survival after LT [6, 7]. However, B-type natriuretic peptide (BNP) has been poorly studied in this context, although its ability to predict mortality in cardiac or septic patients has been investigated [8-10]. Recent studies on the usefulness of serum BNP levels in patients with advanced liver disease reported that the postoperative BNP level was a risk factor predicting deteriorated outcomes after LT, with a suggested postoperative BNP cutoff value of above $400 \mathrm{pg} / \mathrm{mL}$ [11].

BNP is a neurohormone that is secreted mainly from the cardiac ventricles in response to tension of the cardiac wall caused by volume and pressure [12, 13]. Therefore, increased serum BNP levels in patients with cardiac dysfunction have attracted interest to both the diagnostic and prognostic properties of BNP [14]. Serial BNP monitoring is used as a screening test in the intensive care unit to evaluate patients who may develop heart failure and need further evaluation, such as echocardiography, to evaluate the causes of heart failure. In this context, the prognostic ability of BNP levels is worth evaluating in patients undergoing LT, because cardiac death is the leading cause of mortality following LT [15].

In the present study, we evaluated whether BNP had the clinical significance and prognostic ability to predict patient prognosis. We also suggested a new predictive model using BNP by logistic regression analysis of mortality within 1-month of LT, and compared it to pretransplant MELD and MELD-Na scores, which were known to predict mortality after LT.

\section{Material and Methods}

Patients who underwent LT between March 2016 and November 2019 at Seoul St. Mary's Hospital were included in the study. A total of 204 adult patients ( $\geq$ 19 years old) were enrolled in the study. The data were collected retrospectively from our hospital's electronic medical record system. The Institutional Review Board approved the use of the registry data for the patients in our hospital. Owing to the retrospective nature, the requirement for written informed consent to use the data for publication was waived by the Institutional Review Board (KC19RESI0204).

The LT surgical procedures were conducted according to the LT protocol of Seoul St. Mary's Hospital. LT was performed by a piggyback technique using the right hepatic lobes of the donor in living donor LT, and replacing the recipient's retrohepatic vena cava with a donor vena caval segment using end-to-end anastomosis between the graft IVC and the recipient IVC in deceased donor LT. In each case, portal vein and hepatic vein anastomoses were performed first, followed by hepatic artery anastomosis and bile duct reconstruction. A venovenous bypass with a pump was not performed. A temporary surgical portocaval shunt during the anhepatic period to decompress the splanchnic circulation and reduce bowel edema was placed in limited patients with minimal collateral circulation. This was based on preoperative computed tomography findings or a high-pressure gradient $(>5$ $\mathrm{mmHg}$ ) between the portal venous pressure after placement of a catheter at the portal vein and the central venous pressure in the central venous catheter at the internal jugular vein in the perioperative period caused by complete clamping of the portal vein. Histidine-tryptophan-ketoglutarate solution (Custodiol ${ }^{\circledR}$ HTK; Dr. Franz Köhler Chemie GmbH, Bensheim, Germany) was used to preserve the graft livers.

The intraoperative anesthetic management followed our institute's protocol for LT. For anesthesia, balanced anesthesia was provided using $1.5-2 \%$ sevoflurane or $4-6 \%$ desflurane with remifentanil infusion at a rate of $0.1-0.2 \mu \mathrm{g} / \mathrm{kg} / \mathrm{min}$ under guidance by bispectral brain monitoring. Atracurium was continuously administrated at a rate of $6-8 \mu \mathrm{g} / \mathrm{kg} / \mathrm{min}$ for muscle relaxation. After the induction of anesthesia, a Swan-Ganz catheter was inserted into the right internal jugular vein to provide continuous hemodynamic monitoring, including stroke volume, cardiac output, and vascular resistance measurements. For frequent blood sampling and continuous blood pressure monitoring, the radial artery was cannulated and a 22-gauge angiocatheter was placed. The patient hematocrit was maintained between 25 and 30\%, and serum calcium levels and $\mathrm{pH}$ were maintained at $80 \%$ of the lower limit of the normal range $(0.9 \mathrm{mmol} / \mathrm{L})$ of serum ionized calcium [16] and > pH 7.15, respectively, by administering calcium gluconate and sodium bicarbonate with adequate ventilation.

Serial laboratory tests were routinely performed during surgical the phases of LT, which were during the intraoperative period, 60 mins into the pre-anhepatic phase, 30 mins into the anhepatic phase, and 30 mins into the neohepatic phases. Each set of laboratory test included to complete blood count with a differential blood count, blood chemistry with arterial and venous blood gas analysis, tests for disseminated intravascular coagulation, BNP, blood viscosity, and thromboelastogram. Additional laboratory tests were performed at the clinician's discretion. BNP was routinely measured on postoperative day 1 as well as at four time points during the intraoperative period according to the surgical phases (60 mins into the pre-anhepatic phase 
(T1), 30 mins into the anhepatic phase (T2), and 30 mins after reperfusion of the grafted liver (neohepatic phase, T3)) and on postoperative day 1 (T4). Postoperative BNP was routinely measured at postoperative day 1 and additional BNP measurements were performed if the patients had a higher BNP level with simultaneous cardiac diseases. Each BNP sample was collected using the nearest time point within 10 mins of the study time point to minimize confounding by time. The MELD score and MELD-Na score were calculated using previously published formulas [17, 18], and higher MELD and MELD-Na scores indicated more severe hepatic disease.

The primary endpoint was 1-month mortality after LT defined as all-cause mortality during hospitalization. The patients were dichotomized into two groups, survival and non-survival groups.

The variables potentially related to 1-month mortality $(P<0.10)$ were selected using the results from univariate analysis. For calculation of a better prognostic model, multivariate analysis was done. Conditional logistic regression analysis was performed for pairwise interacting variables of two significant BNP time points to create a new prognostic model by combining the BNPs of the T2 and T4, which was called the combined-BNP (cBNP). The BNPs were logarithmically transformed to normal distribution to apply logistic regression. We called the model the combined-BNP (cBNP) model because the model was calculated using two significantly different time points for the BNPs, T2 and T4. The new predictive model was calculated by:

$\mathrm{cBNP}=2.56 \times \mathrm{Ln}$ BNP (anhepatic phase (T2), pg/mL) +2.48

$\times$ Ln BNP (post-operative day 1 (T1), pg/mL) - 28 .

To evaluate the fitness of the cBNP model, the Hosmer-Lemeshow goodness of fit test for logistic regression was performed.

The individual diagnostic accuracy of cBNP, $\mathrm{BNP}, \mathrm{Cr}$ (creatinine), MELD scores, and MELD-Na scores for mortality within 1-month after LT was investigated using the area under the receiver operator characteristic curve (AUROC), and the threshold scores, sensitivities, specificities, positive predictive values, and negative predictive values were calculated. We calculated the discrimination of individual AUROCs using the improvement in individual AUROC models by calculating the difference in the AUROCs ( $\triangle$ AUROC). The AUROCs were compared by a method proposed by DeLong et al. [19].

We calculated the net reclassification improvement (NRI) and integrated discrimination improvement (IDI) to quantify the incremental predictive value between the $\mathrm{BNP}$ and MELD scores from the AUROC analysis resulting in reclassification. The NRI and IDI were calculated by a formula proposed by Pencina et al. [20]. We classified the predicted risk into four strata $(0-5,5-15,15-20$, and > $20 \%$ 1-month mortality risk) for mortality within 1-month after LT.

Statistical analyses were performed using $R$ software version 4.0.2 (R Foundation for Statistical Computing, Vienna, Austria). The study population data are presented as means \pm standard deviations (SD), medians (interquartile ranges, IQR), or absolute values (proportions) as appropriate. All variables were analyzed using parametric or nonparametric tests as appropriate, followed by the KolmogorvSmirnov test to determine the normal distribution of each value. Student's t-tests, or Mann-Whitney test (for continuous variables) and Chi-squared tests, or Fisher's exact test (for categorical variables) were performed to compare the two groups. Conditional logistic regression was done to calculate a new model and the Hosmer-Lemeshow goodness of fit test was performed. The diagnostic accuracy and cutoff value of the models were analyzed using the AUROC. All $P$ values were two-sided, and a $P$ value of $<0.05$ was considered statistically significant.

\section{Results}

Seventeen cases were excluded from this study because they were missing laboratory BNP data needed in the study protocol. Finally, a total of 187 patients were enrolled in the present study (Figure 1). The enrolled patients were dichotomized into two groups in terms of mortality within 1-month after LT, the survival and non-survival groups. Fourteen (7.5 \%) of 187 patients expired within one month after LT and 173 patients survived. The most common cause of mortality was sepsis $(\mathrm{N}=9,64.3 \%)$, followed by vascular complication ( $\mathrm{N}=3,21.4 \%)$, and graft failure $(\mathrm{N}=2,14 \%)$. Of the patients with sepsis, the most common manifestation was pneumonia $(\mathrm{N}=6$, $66.7 \%)$, followed by biliary sepsis $(\mathrm{N}=2,22.2 \%)$, and unknown origin $(\mathrm{N}=1,11.1 \%)$. The vascular complications included hepatic artery thrombosis $(\mathrm{N}$ $=2,66.7 \%)$, and hepatic vein stenosis $(\mathrm{N}=1,33.3 \%)$. Five patients $(35.7 \%)$ of 14 non-surviving patients expired within the first seven days after LT, five (35.7\%) of 14 non-surviving patients expired between 8 and 21 days after LT, and four (28.6\%) of 14 non-surviving patients expired from 22 days to 30 days after LT.

The demographic and preoperative data of the recipients and donors are shown in Table 1. There were more male patients than female patients in both the survival and non-survival groups $(68.8 \%$ males in the survival group vs. $64.3 \%$ in the non-survival 
group). However, there were no significant differences between the two groups. In the scoring system and preoperative laboratory data, the nonsurvival group had higher MELD scores, MELD-Na scores, international normalized ratios (INR), $\mathrm{Cr}$, and $C$-reactive protein than the non-survival group $(P<$ $0.05)$. With respect to the causes of end-stage liver disease (ESLD), there was a significant difference between the two groups $(P<0.05)$. The survival group showed a higher proportion of viral-associated ESLD than non-viral ESLD (viral ESLD, 56.6\%; non-viral ESLD, 43.4\%). However, the difference between viral and non-viral ESLD in the non-survival group was not significantly different (viral ESLD, 50.0\%; non-viral ESLD, 50.0\%). Among the viral-associated ESLD, hepatitis B virus infection was the predominant cause in the survival group $(40.5 \%)$, and hepatitis A virus and hepatocellular carcinoma were the predominant causes in the non-survival group (21.4\% and $21.4 \%$; respectively). With respect to preoperative echocardiography, the ejection fraction (EF) and systolic dysfunction ( $\mathrm{EF}<50 \%$ ) were not significantly different between the two groups. However, there was a higher proportion of patients with systolic dysfunction ( $\mathrm{EF}<50 \%$ ) in the non-survival group than in the survival group $(P=0.220)$. The proportion of patients with diastolic dysfunction and pulmonary hypertension was also not significantly different between the two groups. There were no statistically significant differences between the two groups in demographics and preoperative laboratory data of the recipients and the donors, including age, body mass index, neutrophil-to-lymphocyte (NL) ratio, serum electrolytes, ischemic time of the liver graft, the graft-to-recipient weight ratio (GWRW), and fatty changes in the grafted livers (Table 1 ).

Table 2 shows the perioperative data, including laboratory and hemodynamic parameters according to the three surgical LT phases, pre-anhepatic (T1), anhepatic (T2), and neohepatic phases (T3), and the BNP and neutrophil-to-lymphocyte ratio on postoperative day 1 (T4). The intraoperative and postoperative BNP levels were significantly different between the survival and the non-survival groups in all phases. The BNP levels were higher in the non-survival group than the survival group. The $\mathrm{pH}$ was significantly lower only in the pre-anhepatic phase and the CVP was higher level in the non-survival group than the survival group only in the neohepatic phase $(P<0.05)$. However, the $\mathrm{pH}$ and CVP levels showing a statistical significance between the two groups were within the normal ranges. The creatinine levels, administered fluids, number of patients with ascites $>1 \mathrm{~L}$, and operation time were not significantly different between the two groups, and mean arterial blood pressure (mABP), stroke volume variation (SVV), cardiac index (CI), and systemic vascular resistance index (SVRI) were also not significantly different between the two groups in the perioperative period.

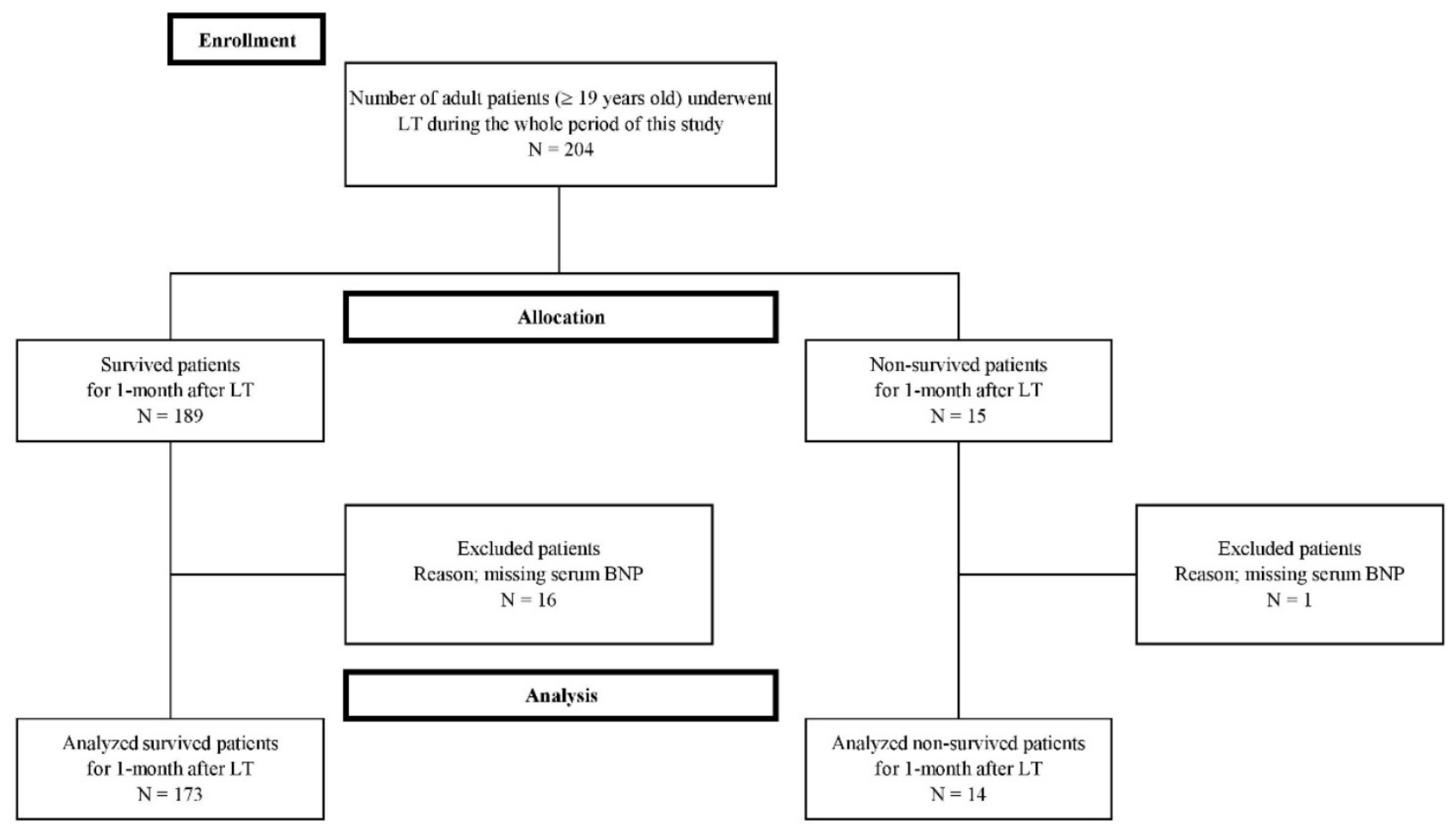

Figure 1. Flow diagram of the study. 
Table 1. Demographic and preoperative data of the study population

\begin{tabular}{|c|c|c|c|}
\hline Characteristic & Survival (N=173) & Non-survival $(\mathrm{N}=14)$ & $P$ value \\
\hline \multicolumn{4}{|l|}{ Recipient } \\
\hline Age (years) & $52 \pm 9$ & $52 \pm 13$ & 0.942 \\
\hline Gender (female/male) & $54(31.2) / 119(68.8)$ & $5(35.7) / 9(64.3)$ & 0.768 \\
\hline \multicolumn{4}{|l|}{ Causes of ESLD } \\
\hline Hepatitis B & $70(40.5)$ & $2(14.3)$ & $0.025^{*}$ \\
\hline Hepatitis C & $23(13.3)$ & $2(14.3)$ & \\
\hline Hepatitis A & $5(2.9)$ & $3(21.4)$ & \\
\hline Alcoholic & $22(12.7)$ & $2(14.3)$ & \\
\hline $\mathrm{HCC}$ & $31(17.9)$ & $3(21.4)$ & \\
\hline Others & $22(12.7)$ & $2(14.3)$ & \\
\hline BMI $\left(\mathrm{kg} / \mathrm{m}^{2}\right)$ & $24.9 \pm 4.0$ & $25.3 \pm 4.5$ & 0.720 \\
\hline MELD (pts) & $18 \pm 11$ & $29 \pm 14$ & $0.017^{*}$ \\
\hline MELD-Na (pts) & $17 \pm 13$ & $27 \pm 17$ & $0.040^{*}$ \\
\hline \multicolumn{4}{|l|}{ Serum } \\
\hline Sodium (mmol/L) & $138.1 \pm 6.0$ & $140.2 \pm 3.7$ & 0.199 \\
\hline Lactate $(\mathrm{mg} / \mathrm{dL})$ & $2.6 \pm 2.5$ & $5.4 \pm 4.5$ & 0.238 \\
\hline bilirubin (total, mg/dL) & $8.0 \pm 11.2$ & $14.0 \pm 15.3$ & 0.172 \\
\hline albumin (mg/dL) & $3.1 \pm 0.6$ & $2.3 \pm 1.0$ & 0.986 \\
\hline AST (U/L) & $167.8 \pm 608.1$ & $745.9 \pm 1555.1$ & 0.190 \\
\hline $\operatorname{ALT}(\mathrm{U} / \mathrm{L})$ & $146.5 \pm 552.1$ & $923.0 \pm 1819.3$ & 0.135 \\
\hline INR & $1.73 \pm 0.77$ & $2.29 \pm 1.04$ & $0.012^{*}$ \\
\hline Creatinine (mg/dL) & $1.22 \pm 1.10$ & $2.15 \pm 1.26$ & $0.003^{*}$ \\
\hline C-RP (mg/L) & $1.39 \pm 2.34$ & $3.14 \pm 2.71$ & $0.009^{*}$ \\
\hline Ejection fraction (\%) & $64.4 \pm 5.0$ & $62.7 \pm 5.1$ & 0.349 \\
\hline Ejection fraction < $50 \%$ & $2(1.2)$ & $1(7.1)$ & 0.220 \\
\hline Diastolic dysfunction & $96(55.5)$ & $11(64.7)$ & 0.093 \\
\hline Pulmonary hypertension & $9(5.2)$ & $2(14.3)$ & 0.120 \\
\hline \multicolumn{4}{|l|}{ Donor } \\
\hline Age (years) & $35 \pm 13$ & $42 \pm 12$ & 0.106 \\
\hline Gender (female/male) & $62(35.8) / 111(64.2)$ & $5(35.7) / 9(64.3)$ & 1.000 \\
\hline Ischemic time (min) & $115 \pm 73$ & $94 \pm 48$ & 0.347 \\
\hline Living / Deceased & $134(77.5) / 39(22.5)$ & $11(78.9) / 3(21.1)$ & 1.000 \\
\hline GWRW & $1.18 \pm 0.45$ & $1.39 \pm 0.56$ & 0.120 \\
\hline Fatty change of graft (\%) & $3.5 \pm 6.1$ & $4.7 \pm 5.1$ & 0.484 \\
\hline
\end{tabular}

Data are presented as mean $\pm \mathrm{SD}$, or numbers (\%).

ESLD, end-stage liver disease; HCC, hepatocellular carcinoma; BMI, body mass index; MELD, model for end-stage liver disease; MELD-Na, sodium conjugated model for end-stage live disease; AST, asparate transaminase; ALT, alanine transaminase; INR, international normalized ratio; GWRW, graft weight to recipient weight.

*Statistically significant differences $(P$ value of $<0.05)$.

Table 2. Laboratory and hemodynamic data according to the three phases of liver transplantation in the survival and the non-survival groups

\begin{tabular}{llll}
\hline Characteristic & Survival $(\mathrm{N}=173)$ & Non-survival $(\mathrm{N}=14)$ & $P$ value \\
\hline BNP $(\mathrm{pg} / \mathrm{mL})$ & & & \\
T1 & $87.6(45.5-171.0)$ & $307.9(174.2-828.1)$ & $<0.001^{*}$ \\
T2 & $79.0(40.5-121.3)$ & $400.5(215.5-445.3)$ & $<0.001^{*}$ \\
T3 & $82.9(41.6-128.3)$ & $216.2(189.0-291.0)$ & $<0.001^{*}$ \\
T4 & $106.0(73.0-127.0)$ & $214.5(174.7-242.8)$ & $<0.001^{*}$ \\
Creatinine (mg/dL) & & & \\
T1 & $1.11 \pm 0.48$ & $1.56 \pm 1.38$ & 0.249 \\
T2 & $1.28 \pm 0.81$ & $1.15 \pm 0.33$ & 0.549 \\
T3 & $1.19 \pm 0.65$ & $1.00 \pm 0.11$ & 0.255 \\
Glucose (mg/dL) & & & \\
T1 & $142 \pm 42.0$ & $113 \pm 46.0$ & 0.697 \\
T2 & $154 \pm 51.0$ & $140 \pm 52.0$ & 0.382 \\
T3 & $208 \pm 51.0$ & $183 \pm 49.0$ & 0.167 \\
Neutrophile-to-Lymphocyte ratio & & \\
T1 & $2.90(1.57-5.92)$ & $3.47(2.51-11.28)$ & 0.119 \\
T2 & $7.67(4.68-12.43)$ & $9.88(1.93-11.65)$ & 0.606 \\
T3 & $11.17(7.31-16.75)$ & $12.99(1.94-18.29)$ & 0.625 \\
T4 & $17.42(10.67-24.37)$ & $14.30(8.29-19.97)$ & 0.223 \\
\hline
\end{tabular}

\begin{tabular}{|c|c|c|c|}
\hline Characteristic & Survival $(\mathrm{N}=173)$ & Non-survival (N=14) & $P$ value \\
\hline \multicolumn{4}{|l|}{$\mathrm{pH}$} \\
\hline $\mathrm{T} 1$ & $7.40 \pm 0.07$ & $7.33 \pm 0.09$ & $<0.001^{*}$ \\
\hline $\mathrm{T} 2$ & $7.28 \pm 0.10$ & $7.24 \pm 0.12$ & 0.158 \\
\hline T3 & $7.30 \pm 0.08$ & $7.28 \pm 0.11$ & 0.263 \\
\hline \multicolumn{4}{|l|}{ Lactate $(\mathrm{mg} / \mathrm{dL})$} \\
\hline $\mathrm{T} 1$ & $2.60 \pm 3.50$ & $3.80 \pm 4.16$ & 0.225 \\
\hline $\mathrm{T} 2$ & $5.82 \pm 3.05$ & $6.13 \pm 3.78$ & 0.745 \\
\hline T3 & $5.72 \pm 3.39$ & $5.55 \pm 3.39$ & 0.861 \\
\hline \multicolumn{4}{|c|}{ Transfusion PRBCs (unit) } \\
\hline $\mathrm{T} 1$ & $3.8 \pm 4.4$ & $6.9 \pm 7.2$ & 0.144 \\
\hline T2 & $2.8 \pm 4.0$ & $2.8 \pm 2.3$ & 0.959 \\
\hline T3 & $3.1 \pm 3.4$ & $3.8 \pm 3.8$ & 0.525 \\
\hline All periods & $9.9 \pm 9.1$ & $13.6 \pm 7.1$ & 0.083 \\
\hline \multicolumn{4}{|l|}{ Administered fluids } \\
\hline Crystalloids (L) & $6.8 \pm 3.4$ & $6.4 \pm 2.7$ & 0.678 \\
\hline Colloids (mL) & $805 \pm 476$ & $746 \pm 552$ & 0.664 \\
\hline Ascites $>1 \mathrm{~L}$ & $79(45.7)$ & $8(57.1)$ & 0.408 \\
\hline \multicolumn{4}{|l|}{ Hemodynamics } \\
\hline \multicolumn{4}{|l|}{ HR (beat/min) } \\
\hline $\mathrm{T} 1$ & $84 \pm 16$ & $91 \pm 17$ & 0.125 \\
\hline $\mathrm{T} 2$ & $94 \pm 18$ & $95 \pm 23$ & 0.953 \\
\hline $\mathrm{T} 3$ & $95 \pm 14$ & $95 \pm 20$ & 0.937 \\
\hline \multicolumn{4}{|l|}{ mABP (mmHg) } \\
\hline $\mathrm{T} 1$ & $76 \pm 14$ & $81 \pm 14$ & 0.583 \\
\hline $\mathrm{T} 2$ & $73 \pm 14$ & $79 \pm 12$ & 0.055 \\
\hline $\mathrm{T} 3$ & $76 \pm 47$ & $74 \pm 12$ & 0.833 \\
\hline \multicolumn{4}{|l|}{ CVP (mmHg) } \\
\hline $\mathrm{T} 1$ & $10 \pm 4$ & $11 \pm 5$ & 0.374 \\
\hline $\mathrm{T} 2$ & $9 \pm 4$ & $10 \pm 4$ & 0.393 \\
\hline T3 & $11 \pm 4$ & $14 \pm 4$ & $0.026^{*}$ \\
\hline \multicolumn{4}{|l|}{ SVV (\%) } \\
\hline $\mathrm{T} 1$ & $7.3 \pm 3.9$ & $5.3 \pm 2.1$ & 0.253 \\
\hline $\mathrm{T} 2$ & $9.2 \pm 8.0$ & $7.8 \pm 4.7$ & 0.595 \\
\hline $\mathrm{T} 3$ & $6.7 \pm 4.1$ & $6.9 \pm 4.5$ & 0.854 \\
\hline \multicolumn{4}{|l|}{$\mathrm{CI}\left(\mathrm{L} / \mathrm{min} / \mathrm{m}^{2}\right)$} \\
\hline $\mathrm{T} 1$ & $4.0 \pm 1.1$ & $4.1 \pm 0.7$ & 0.349 \\
\hline $\mathrm{T} 2$ & $3.8 \pm 1.2$ & $4.1 \pm 1.2$ & 0.644 \\
\hline $\mathrm{T} 3$ & $4.8 \pm 1.3$ & $4.7 \pm 1.3$ & 0.697 \\
\hline \multicolumn{4}{|c|}{ SVRI (dynes-sec/cm-5/m²) } \\
\hline $\mathrm{T} 1$ & $1338 \pm 453$ & $1334 \pm 386$ & 0.891 \\
\hline $\mathrm{T} 2$ & $1404 \pm 545$ & $1505 \pm 859$ & 0.626 \\
\hline $\mathrm{T} 3$ & $1077 \pm 417$ & $1054 \pm 445$ & 0.881 \\
\hline Operation time (hr) & $8.6 \pm 1.5$ & $8.4 \pm 2.1$ & 0.569 \\
\hline \multicolumn{4}{|c|}{ Data are presented as mean \pm SD or numbers $(\%)$. } \\
\hline \multicolumn{4}{|c|}{$\begin{array}{l}\mathrm{T} 1,60 \text { mins into pre-anhepatic phase; T2, } 30 \text { mins into anhepatic phase; } \mathrm{T} 3,30 \mathrm{~min} \\
\text { after reperfusion of the grafted liver (neohepatic phase); T4, postoperative day } 1 .\end{array}$} \\
\hline
\end{tabular}

The BNP values were log-transformed due to the skewness of the BNP value distribution and multivariate adjustment was done using the results from the univariate analysis of the BNPs in logistic regression. Table 3 shows that the 1-month mortality increased 12.9 and 12.0 times when the LN BNPs at T2 and T4 increased by one point, respectively. We calculated the cBNP using the results from conditional logistic regression and evaluated the goodness of fit of the regression model using the Hosmer and Lemeshow test. The Hosmer and Lemeshow test showed a good fit of the cBNP model with a $X^{2}$ and $P$ value of 1.24 and 0.996 , respectively [21].

We compared the predictive ability of cBNP, 
each BNP time point, the MELD scores, the MELD-Na scores for 1-month mortality using the AUROC (Table $4)$. All of them had statistical significance in predicting 1 -month mortality after LT $(P<0.05)$. The cBNP was significantly different from the MELD scores, and the MELD-Na scores from the AUROC analyses ( $\triangle$ AUROC 0.262, and 0.286, $P=0.001$, and $P<0.001$, respectively). The cBNP showed the highest predictive accuracy, and the BNP value at postoperative day 1 (T4) had the next best accuracy predicting 1-month mortality (95\% CI: 0.941-0.992; AUROC $=0.976$ and 95\% CI: 0.925-0.985; AUROC $=$ 0.963, respectively). The most discriminatory cutoff values for short-term mortality within 1-month after LT determined using AUROC analyses were an LN BNP level at T2 of $>4.942$ and an LN BNP at T4 of $>$ 5.228 for the cBNP, and a BNP at T2 of $>175 \mathrm{pg} / \mathrm{mL}$, a BNP at T4 of $>155 \mathrm{pg} / \mathrm{mL}$, a MELD score $>31$, and a MELD-Na score $>29$. LN BNP values of 4.942 and 5.228 were equivalent to BNP values of $137 \mathrm{pg} / \mathrm{mL}$, and $187 \mathrm{pg} / \mathrm{mL}$, respectively. At this cutoff point, the cBNP and MELD scores showed $100 \%$ and $57.1 \%$ sensitivity, and $91.9 \%$ and $85.9 \%$ specificity, respectively. Figure 2 shows AUROCs and 95\% CIs for the cBNP, MELD scores, and MELD-Na scores.

Table 3. Multivariate analysis including the odds ratio of the BNP and $\mathrm{Cr}$ at time points between the survival and the non-survival groups

\begin{tabular}{|c|c|c|c|c|c|}
\hline & Survival (N=173) & $\begin{array}{l}\text { Non-survival } \\
(\mathrm{N}=14)\end{array}$ & $P$ value & Odds ratio $(95 \% \mathrm{CI})$ & $\begin{array}{l}P \\
\text { value }\end{array}$ \\
\hline \multicolumn{6}{|c|}{$\mathrm{BNP}(\mathrm{pg} / \mathrm{mL})$} \\
\hline $\mathrm{T} 1$ & $87.6(45.5-171.0)$ & $307.9(174.2-828.1)$ & $<0.001^{*}$ & & \\
\hline $\mathrm{T} 2$ & $79.0(40.5-121.3)$ & $400.5(215.5-445.3)$ & $<0.001^{*}$ & $1.010(1.000-1.020)$ & 0.003 \\
\hline $\mathrm{T} 3$ & 82.9 (41.6-128.3) & $216.2(189.0-291.0)$ & $<0.001^{*}$ & & \\
\hline $\mathrm{T} 4$ & $106.0(73.0-127.0)$ & $214.5(174.7-242.8)$ & $<0.001^{*}$ & $1.011(1.001-1.026)$ & 0.010 \\
\hline \multicolumn{6}{|c|}{ LN BNP } \\
\hline $\mathrm{T} 1$ & $4.44 \pm 1.00$ & $5.88 \pm 0.78$ & $<0.001^{*}$ & & \\
\hline $\mathrm{T} 2$ & $4.25 \pm 0.82$ & $5.81 \pm 0.44$ & $<0.001^{*}$ & $12.9(2.349-70.963)$ & 0.003 \\
\hline $\mathrm{T} 3$ & $4.28 \pm 0.88$ & $5.46 \pm 0.29$ & $<0.001^{*}$ & & \\
\hline T4 & $4.55 \pm 0.51$ & $5.36 \pm 0.21$ & $<0.001^{*}$ & $12.0(1.006-142.759)$ & 0.049 \\
\hline \multicolumn{6}{|c|}{$\begin{array}{l}\text { LN BNP; log-transformation of the BNP levels at each time point; BNP, } \\
\text { B-natriuretic peptide; CI, confidence interval. }\end{array}$} \\
\hline \multicolumn{6}{|c|}{$\begin{array}{l}\mathrm{T} 1,60 \text { mins into pre-anhepatic phase; } \mathrm{T} 2,30 \mathrm{mins} \text { into anhepatic phase; } \mathrm{T} 3,30 \mathrm{mins} \\
\text { after reperfusion of the grafted liver (neohepatic phase); T4, postoperative day } 1 .\end{array}$} \\
\hline
\end{tabular}

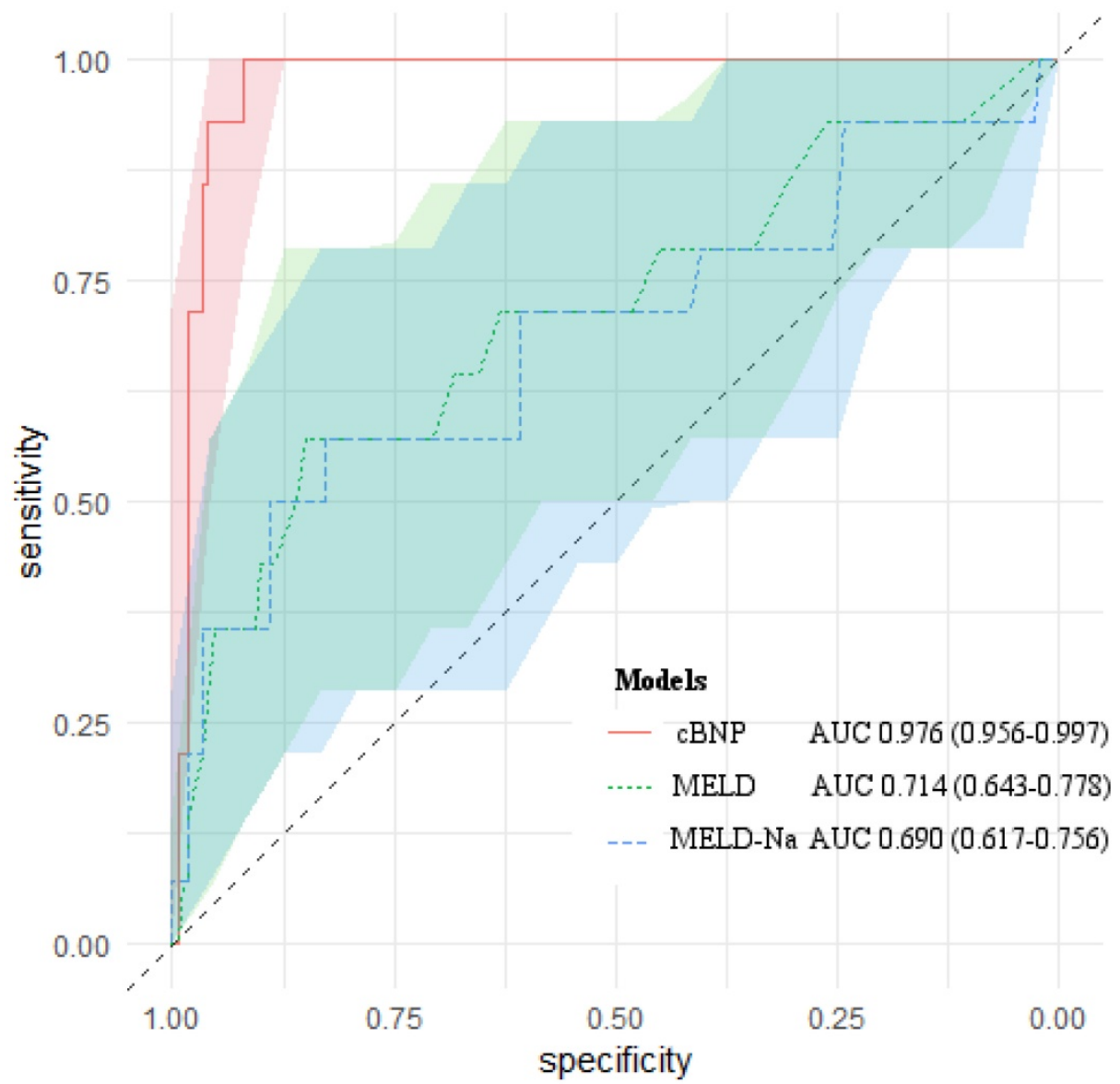

Figure 2. AUROCs of CBNP, MELD scores and MELD-Na scores for predicting 1-month mortality after liver transplantation. The light-colored areas indicate $95 \%$ confidence regions for the AUROCs. 
Table 4. Comparison of the predictive values, sensitivity, specificity, diagnostic accuracy, and differences in AUROCs of the cBNP, BNP, $\mathrm{Cr}$, MELD scores, and MELD-Na scores for predicting 1-month mortality after liver transplantation

\begin{tabular}{|c|c|c|c|c|c|c|c|c|c|}
\hline Prognostic test & Threshold & Sensitivity & Specificity & PPV & NPV & Diagnostic accuracy & $\triangle \mathrm{AUROC}$ & $95 \% \mathrm{CI}$ & $P$ value \\
\hline \multicolumn{10}{|l|}{ cBNP score } \\
\hline \multicolumn{10}{|l|}{ BNP $(\mathrm{pg} / \mathrm{mL})$} \\
\hline $\mathrm{T} 2$ & $>137$ & 100.0 & 91.9 & 50.0 & 100.0 & 0.976 & Ref. & 0.94-0.99 & Ref. \\
\hline $\mathrm{T} 4$ & $>187$ & & & & & & & & \\
\hline MELD (pts) & $>31$ & 57.1 & 85.9 & 25.0 & 96.1 & 0.714 & 0.262 & $0.64-0.78$ & $0.001^{*}$ \\
\hline MELD-Na (pts) & $>29$ & 57.1 & 82.9 & 21.6 & 95.9 & 0.690 & 0.286 & $0.62-0.76$ & $<0.001^{*}$ \\
\hline \multicolumn{10}{|l|}{ BNP (pg/mL) } \\
\hline $\mathrm{T} 1$ & $>124$ & 100.0 & 64.7 & 18.7 & 100.0 & 0.865 & 0.111 & $0.81-0.91$ & $0.021^{*}$ \\
\hline $\mathrm{T} 2$ & $>175$ & 92.9 & 89.6 & 41.9 & 99.4 & 0.962 & 0.015 & $0.92-0.99$ & 0.545 \\
\hline T3 & $>150$ & 100.0 & 80.3 & 29.2 & 100.0 & 0.913 & 0.063 & 0.86-0.95 & 0.210 \\
\hline $\mathrm{T} 4$ & $>155$ & 100.0 & 94.2 & 58.3 & 100.0 & 0.963 & 0.013 & 0.93-0.99 & 0.723 \\
\hline
\end{tabular}

BNP, B-type natriuretic peptide; cBNP, combined BNP; MELD, model for end-stage liver disease; MELD-Na, sodium conjugated model for end-stage live disease; PPV, positive predictive value; NPV, negative predictive value; AUROC, area under the receiver operating characteristic curve; $\triangle A U R O C$, difference in AUROCs.

T1, 60 mins into pre-anhepatic phase; T2, 30 mins into anhepatic phase; T3, 30 mins after reperfusion of the grafted liver (neohepatic phase); T4, post-operative day 1. a $P$ value calculated for the comparison of cBNP vs. the other models.

*Statistically significant differences $(P$ value of $<0.05)$.

Table 5. Reclassification of predicted of 1 -month mortality risk after LT between the cBNP and MELD scoring systems

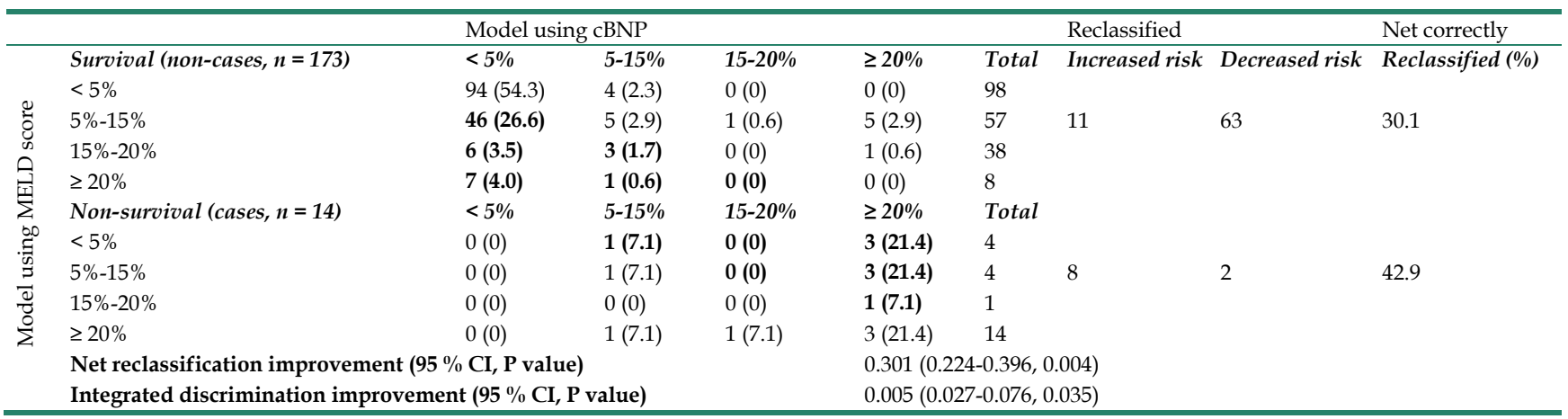

Table 5 summarizes the results from the reclassification of the individual models using the cBNP values and MELD scores. Eleven individuals who survived for 1-month after LT were reclassified up and 63 individuals were reclassified down. It improved the net gain with a reclassification proportion of 0.301 . Of the patients who did not survive one month after LT, eight were reclassified up and two were reclassified down. It improved the net gain in the reclassification proportion to 0.429 . Therefore, the NRI was estimated at 0.301 (95\%CI: $0.224-0.396)$ and was significantly different $(P=0.004)$. The IDI was estimated at 0.005 (95\%CI: 0.027-0.076) and was also significant $(P=0.035)$.

\section{Discussion}

We proposed $\mathrm{cBNP}$ as a new predictive BNP model that showed better diagnostic accuracy than the other models using BNP levels at single time points, the MELD score, or the MELD-Na score to predict mortality within 1-month for all causes after LT. Our results showed that the BNP level at a single time point was also suitable for predicting the mortality in patients who underwent LT. However, the combination of BNP levels from two different time points showed improved predictive accuracy for 1-month mortality in patients who underwent LT compared to BNP values at single time points. Moreover, cBNP had better predictive value than MELD scores, even if the MELD score was created to predict the severity of hepatic disease in patients with ESLD [22-24].

Several studies have reported predictive models for mortality after LT, including pretransplant MELD scores, and MELD-Na scores [4, 25-28]. The MELD scores well-known useful predictors of early mortality after LT, with higher MELD scores associated with higher mortality, although the MELD score was designed to estimate the survival in patients with elective placement of a trans-jugular intrahepatic portosystemic shunt for portal hypertension [29, 30]. Therefore, we tried to compare the serum BNP levels and a new predictive model to the MELD score, which is a representative predictor related to the mortality in patients undergoing LT, to confirm the predictive ability of the suggested a new model.

BNP is secreted from the ventricular muscle of the heart into the circulation in response to tension stress on the cardiac wall and has a half-life in the circulation of about 20 mins [31-33]. BNP levels have 
been used to predict the mortality and morbidity of patients with cardiac diseases, with higher BNP levels correlating with greater risk [34-36]. A cutoff value above $100 \mathrm{pg} / \mathrm{ml}$ BNP was recommended for a diagnosis of heart failure [37, 38]. However, the usefulness of BNP levels related to mortality after LT in patients with ESLD has been poorly evaluated. Two studies have been conducted on the relationship between BNP and the patients with liver disease. One reported that $\mathrm{BNP}$ was related to the severity of liver cirrhosis in non-alcoholic patients. The study demonstrated that the patients with higher BNP levels showed poor Child-Pugh classification and more advanced cirrhosis [39]. The other evaluated whether pretransplant and posttransplant BNP could predict mortality after LT [11]. The study reported that posttransplant BNP was associated with mortality and poor outcomes after LT. The suggested cutoff value of $\mathrm{BNP}$ on posttranplant day 3 was $>400$ $\mathrm{pg} / \mathrm{mL}$. We evaluated the usefulness of BNP to predict mortality after LT using MELD scores instead of the Child-Pugh classification, and BNP levels in intraoperative period and on postoperative day 1 . Our results showed that values above 120 to $190 \mathrm{pg} / \mathrm{mL}$ were cutoff values for perioperative and postoperative day $1 \mathrm{BNP}$ levels, for the diagnosis of mortality within one month after LT.

Several studies have reported elevated BNP levels in patients with ESLD. Elevated BNP levels in patients with cirrhotic liver disease were used to explained cirrhotic cardiomyopathy and silent heart failure. Some patients showed elevated BNP levels in patients with EFs within the normal range [40] because BNP is a marker of early-stage heart disease. Thus, the following reasons for the relationship between BNP levels and the severity of ESLD were suggested. ESLD leads to hyperdynamic syndrome in the cardiovascular system, which is a pathophysiology of ESLD [41, 42]. It is characterized by increased heart rate and cardiac output and decreased systemic vascular resistance with normal or low blood arterial pressure [43]. Increased cardiac performance to maintain appropriate systemic circulation promotes heart wall injury and myocardiocyte stretching, which release BNP into the circulation [44]. Therefore, patients with more advanced cirrhosis would have higher levels of BNP in the circulation. In our study, there was also no significant difference in the preoperative $\mathrm{EF}$ and diastolic dysfunction because BNP levels could be increased even in the early stage of cardiomyopathy as in asymptomatic patients with cirrhotic cardiomyopathy or silent heart failure [45]. The BNP levels in the perioperative period and on postoperative day 1 were higher in the non-survival group than in the survival group and each BNP levels had a significant predictive ability for 1-month mortality after LT in our study. However, cardiac function, intravascular volume status and volume responsiveness such as EF, CVP, SVV, administered fluids, transfused PRBCs, and the numbers of patients with ascites $>1 \mathrm{~L}$ showed no significant difference. With respect to cardiac function, the proportion of patients with systolic (EF < 50\%) and diastolic dysfunction and pulmonary hypertension was higher in the non-survival group than in the survival group although it was not significantly different between the two groups. Thus, asymptomatic cirrhotic cardiomyopathy was associated with elevated BNP levels in patients with ESLD.

The BNP levels at the four time points were significantly different between the survival and non-survival groups in this study (Table 3; $P<0.001$ ). All of them had significant predictive abilities for 1-month mortality after LT. We investigated a better predictive model by calculating the BNPs at different time points, even if BNP levels at each individual time point had excellent diagnostic accuracy (Table 4; all of AUROCs > 0.85). We investigated a better predictive model by combining the values at each BNP time point. From the result of conditional logistic regression, the cBNP had an outstanding diagnostic accuracy compared to the BNP levels at single time points, the MELD score, or the MELD-Na score (cBNP AUROC $=0.976$ ). The BNP levels at $\mathrm{T} 2$ and $\mathrm{T} 4$ were selected, and the BNP levels at $\mathrm{T} 1$ and $\mathrm{T} 3$ were excluded from the cBNP model by multivariate analysis. We assumed that the reason for the multivariate analysis results was the characteristics of each surgical period. The hemodynamic characteristics at $\mathrm{T} 2$ and $\mathrm{T} 4$ had more instability than those at the T1 and T3 points. T1 in the pre-anhepatic phase was when the dissection of the recipient's liver was performed. Thus, the period was accompanied by a rapid blood loss and fluid shift due to varices and adhesion in the abdomen and ascitic decompression. T3 in neohepatic phase was performed the reperfusion of the preserved liver. Thus, the period had a massive release of cold, hyperkalemic acidotic fluid into the recipient's circulation [46]. Therefore, we assumed that the BNP levels at T2 and T4 were selected by multivariate analysis because those at $\mathrm{T} 1$ and T3 would be inappropriate due to hemodynamic instability in the systemic circulation.

The NRI and IDI were calculated to identify diagnostic improvement in using cBNP as a new predictive model compared to the MELD scores. The use of cBNP improved the predictive accuracy of mortality within 1-month after LT 30.1\% compared to the MELD score. The IDI also confirmed 0.5\% 
improved diagnostic accuracy of the cBNP (the NRI and IDI between the cBNP and MELD scores; $30.1 \%$ and $0.5 \% ; P=0.004$, and 0.035 , respectively).

We determined the optimal cutoff for the cBNP, BNPs, MELD scores, MELD-Na scores with the best predictive accuracy for mortality within 1-month after LT using AUROC analysis. Using a cBNP cutoff of T2 BNP levels of $>137 \mathrm{pg} / \mathrm{mL}$, and T4 BNP levels of $>$ $187 \mathrm{pg} / \mathrm{mL}$, the sensitivity and specificity of cBNP were as high as $100 \%$ and $91.9 \%$, respectively, although the AUROC of cBNP was not significantly different from the AUROC of the BNP levels at single time points except for the T1 BNP levels. Only BNP levels at T1, the MELD score, and the MELD-Na score had significantly different AUROCs. BNP levels at postoperative day 1 (T4) were the second-best predictor and showed sensitivity and specificity as high as $100 \%$ and $94.2 \%$, respectively, using a cutoff of 155. The difference in diagnostic accuracy between the cBNP and BNP levels at T4 was 0.013 and the sensitivity and specificity were $100 \%$ and $>90 \%$, respectively, both for cBNP and BNP levels at $\mathrm{T} 4$. Thus, cBNP can be especially useful to clinicians in predicting whether a patient undergoing LT will have a high risk for mortality after transplant (Table 4). BNP levels at T4 are also useful to clinicians, if BNP levels at two time points are not available.

There were several limitations to the interpretation of the results in this study. First, the clinical heterogeneity of the causes of mortality was not adjusted in the study. Second, we were unable to identify pathogenic mechanisms linking cardiac dysfunction, serum BNP levels, and the severity of liver disease. Only the preoperative EF and systolic and diastolic dysfunction by echocardiography were adjusted for cardiac dysfunction. There were limited variables for cardiac evaluation due to the retrospective nature of the study. Third, the use of diuretics and beta-blockers in patients with advanced liver diseases and hypertension have biased our results by underestimating cardiac alterations in patients with advanced liver disease. Fourth, the majority of ESLD in the study population was caused by hepatitis B virus infection, which is a predominant cause of ESLD in Asia. Finally, there are no established guidelines for treating patients with elevated BNP levels, and it is hypothesized that the severity of advanced liver diseases is correlated with elevated levels of serum BNP.

In conclusion, our findings support the outstanding prognostic power of cBNP and its usefulness as a predictive model of patient mortality within one month of LT. The diagnostic accuracy of cBNP was increased by as much as $30.1 \%$ compared to the MELD scores based on the results of the NRI, IDI, and AUROCs. We evaluated the prognostic ability of BNP levels in patients undergoing LT and found that it could provide helpful information to transplant clinicians for predicting patient mortality and providing the best medical treatment. These putative roles require further investigation in a larger, prospective randomized control study, as well as the external validation of our suggested cBNP model. Further studies should be conducted to explore whether potential interventions such as the use of diuretics and beta-blockers could reduce BNP levels and further improve patient prognosis.

\section{Abbreviations}

AUROC: area under the receiver operating characteristic curve; BMI: body mass index; BNP: B-type natriuretic peptide; cBNP: combined B-type natriuretic peptide; ESLD: end-stage liver disease; EF: ejection fraction; LT: liver transplantation; MELD: model for end-stage liver disease; MELD-Na: incorporated sodium into MELD; GWRW: graft weight to recipient weight.

\section{Authorship}

- Conceptualization: Hyun Sik Chung, Yun Sung Jo

- Data curation: Hyun Sik Chung, AMi Woo

- Formal analysis: Hyun Sik Chung, MinSuk Chae, Sang Hyun Hong, Chul Soo Park

- Investigation: Hyun Sik Chung, AMi Woo

- Methodology: Hyun Sik Chung, Jong Ho Choi

- Project administration: MinSuk Chae, Sang Hyun Hong, Chul Soo Park

- Writing - original draft: Hyun Sik Chung

- Writing - review \& editing: Jong Ho Choi, Yun Sung Jo.

\section{Competing Interests}

The authors have declared that no competing interest exists.

\section{References}

1. Pan HC, Jenq CC, Lee WC, et al. Scoring systems for predicting mortality after liver transplantation. PLoS One 2014; 9(9): e107138.

2. Wehler M, Kokoska J, Reulbach U, Hahn EG, Strauss R. Short-term prognosis in critically ill patients with cirrhosis assessed by prognostic scoring systems. Hepatology 2001; 34(2): 255-61.

3. Pan HC, Jenq CC, Tsai MH, et al. Scoring systems for 6-month mortality in critically ill cirrhotic patients: a prospective analysis of chronic liver failure sequential organ failure assessment score (CLIF-SOFA). Aliment Pharmacol Ther. 2014; 40(9): 1056-65.

4. Habib S, Berk B, Chang CC, et al. MELD and prediction of post-liver transplantation survival. Liver Transpl. 2006; 12(3): 440-7.

5. Pan HC, Chen YJ, Lin JP, et al. Proteinuria can predict prognosis after liver transplantation. BMC Surg. 2016; 16(1): 63.

6. Coss E, Watt KD, Pedersen R, Dierkhising R, Heimbach JK, Charlton MR. Predictors of cardiovascular events after liver transplantation: a role for pretransplant serum troponin levels. Liver Transpl. 2011; 17(1): 23-31. 
7. Watt KD, Coss E, Pedersen RA, Dierkhising R, Heimbach JK, Charlton MR Pretransplant serum troponin levels are highly predictive of patient and graft survival following liver transplantation. Liver Transpl. 2010; 16(8): 990-8.

8. Gioli-Pereira L, Marcondes-Braga FG, Bernardez-Pereira S, et al. Predictors of one-year outcomes in chronic heart failure: the portrait of a middle income country. BMC Cardiovasc Disord. 2019; 19(1): 251.

9. Pandompatam G, Kashani K, Vallabhajosyula S. The role of natriuretic peptides in the management, outcomes and prognosis of sepsis and septic shock. Rev Bras Ter Intensiva. 2019; 31(3): 368-78.

10. Hwang GS. B-type natriuretic peptide in anesthesia practice to predict adverse cardiovascular outcomes. Korean J Anesthesiol. 2011; 61(1): 1-2

11. Kwon HM, Moon YJ, Kim KS, et al. Prognostic value of B-type natriuretic peptide in liver transplant patients: Implication in post-transplant mortality. Hepatology 2020; [Epub ahead of print]

12. Tsutamoto T, Wada A, Maeda K, et al. Attenuation of compensation of endogenous cardiac natriuretic peptide system in chronic heart failure: prognostic role of plasma brain natriuretic peptide concentration in patients with chronic symptomatic left ventricular dysfunction. Circulation 1997; 96(2): 509-16.

13. Chen $\mathrm{HH}$, Burnett JC. Natriuretic peptides in the pathophysiology of congestive heart failure. Curr Cardiol Rep. 2000; 2(3): 198-205.

14. Boldanova $T$, Noveanu M, Breidthardt $T$, et al. Impact of history of heart failure on diagnostic and prognostic value of BNP: results from the B-type Natriuretic Peptide for Acute Shortness of Breath Evaluation (BASEL) study. Int J Cardiol. 2010; 142(3): 265-72.

15. Koshy AN, Gow PJ, Han HC, et al. Sudden cardiac death following liver transplantation: Incidence, trends and risk predictors. Int J Cardiol. 2021; 327: 171-4.

16. Forman DT, Lorenzo L. Ionized calcium: its significance and clinical usefulness. Ann Clin Lab Sci. 1991; 21(5): 297-304.

17. Kamath PS, Wiesner RH, Malinchoc M, et al. A model to predict survival in patients with end-stage liver disease. Hepatology 2001; 33(2): 464-70.

18. Biggins SW, Rodriguez HJ, Bacchetti P, Bass NM, Roberts JP, Terrault NA. Serum sodium predicts mortality in patients listed for liver transplantation. Hepatology 2005; 41(1): 32-9.

19. DeLong ER, DeLong DM, Clarke-Pearson DL. Comparing the areas under two or more correlated receiver operating characteristic curves: a nonparametric approach. Biometrics 1988; 44(3): 837-45.

20. Pencina MJ, D'Agostino RB, Sr., D'Agostino RB, Jr., Vasan RS. Evaluating the added predictive ability of a new marker: from area under the ROC curve to reclassification and beyond. Stat Med. 2008; 27(2): 157-72; discussion 207-12.

21. Hosmer DW, Hjort NL. Goodness-of-fit processes for logistic regression: simulation results. Stat Med. 2002; 21(18): 2723-38.

22. Botta F, Giannini E, Romagnoli P, et al. MELD scoring system is useful for predicting prognosis in patients with liver cirrhosis and is correlated with residual liver function: a European study. Gut 2003; 52(1): 134-9.

23. Lau T, Ahmad J. Clinical applications of the Model for End-Stage Liver Disease (MELD) in hepatic medicine. Hepat Med. 2013; 5: 1-10.

24. Saab S, Landaverde $\mathrm{C}$, Ibrahim AB, et al. The MELD score in advanced liver disease: association with clinical portal hypertension and mortality. Exp Clin Transplant. 2006; 4(1): 395-9.

25. Olivari D, Mainardi V, Rando $\mathrm{K}$, et al. Risk Factors of Mortality After Liver Transplantation in Uruguay. Transplant Proc. 2018; 50(2): 499-502.

26. Elkholy S, Mogawer S, Hosny A, et al. Predictors of Mortality in Living Donor Liver Transplantation. Transplant Proc. 2017; 49(6): 1376-82.

27. Ragonete Dos Anjos Agostini AP, de Fatima Santana Boin I, Marques Tonella $\mathrm{R}$, et al. Mortality Predictors After Liver Transplant in the Intensive Care Unit. Transplant Proc. 2018; 50(5): 1424-7.

28. Acharya G, Kaushik RM, Gupta R, Kaushik R. Child-Turcotte-Pugh Score, MELD Score and MELD-Na Score as Predictors of Short-Term Mortality among Patients with End-Stage Liver Disease in Northern India. Inflamm Intest Dis. 2020; 5(1): 1-10.

29. Kamath PS, Kim WR. The model for end-stage liver disease (MELD). Hepatology 2007; 45(3): 797-805.

30. Malinchoc M, Kamath PS, Gordon FD, Peine CJ, Rank J, ter Borg PC. A model to predict poor survival in patients undergoing transjugular intrahepatic portosystemic shunts. Hepatology 2000; 31(4): 864-71.

31. Levin ER, Gardner DG, Samson WK. Natriuretic peptides. N Engl J Med. 1998; 339(5): 321-8.

32. Mukoyama M, Nakao K, Saito $Y$, et al. Increased human brain natriuretic peptide in congestive heart failure. N Engl J Med. 1990; 323(11): 757-8.

33. Yamamoto K, Burnett JC, Jr., Jougasaki M, et al. Superiority of brain natriuretic peptide as a hormonal marker of ventricular systolic and diastolic dysfunction and ventricular hypertrophy. Hypertension 1996; 28(6): 988-94.

34. Santaguida PL, Don-Wauchope AC, Oremus M, et al. BNP and NT-proBNP as prognostic markers in persons with acute decompensated heart failure: a systematic review. Heart Fail Rev. 2014; 19(4): 453-70.

35. Wolsk E, Claggett B, Pfeffer MA, et al. Role of B-Type Natriuretic Peptide and N-Terminal Prohormone BNP as Predictors of Cardiovascular Morbidity and Mortality in Patients With a Recent Coronary Event and Type 2 Diabetes Mellitus. J Am Heart Assoc. 2017; 6(6): e004743.

36. York MK, Gupta DK, Reynolds CF, et al. B-Type Natriuretic Peptide Levels and Mortality in Patients With and Without Heart Failure. J Am Coll Cardiol. 2018; 71(19): 2079-88.
37. Mehra MR, Maisel A. B-type natriuretic peptide in heart failure: diagnostic, prognostic, and therapeutic use. Crit Pathw Cardiol. 2005; 4(1): 10-20.

38. Maisel A. B-type natriuretic peptide levels: diagnostic and prognostic in congestive heart failure: what's next? Circulation 2002; 105(20): 2328-31.

39. Yildiz R, Yildirim B, Karincaoglu M, Harputluoglu M, Hilmioglu F. Brain natriuretic peptide and severity of disease in non-alcoholic cirrhotic patients. J Gastroenterol Hepatol. 2005; 20(7): 1115-20.

40. Zardi EM, Zardi DM, Chin D, Sonnino C, Dobrina A, Abbate A. Cirrhotic cardiomyopathy in the pre- and post-liver transplantation phase. J Cardiol. 2016; 67(2): 125-30.

41. Møller S, Bendtsen F. The pathophysiology of arterial vasodilatation and hyperdynamic circulation in cirrhosis. Liver Int. 2018; 38(4): 570-80.

42. Licata A, Mazzola A, Ingrassia D, Calvaruso V, Cammà C, Craxì A. Clinical implications of the hyperdynamic syndrome in cirrhosis. Eur J Intern Med. 2014; 25(9): 795-802.

43. Zardi EM, Abbate A, Zardi DM, et al. Cirrhotic cardiomyopathy. J Am Coll Cardiol. 2010; 56(7): 539-49.

44. de Lemos JA, McGuire DK, Drazner MH. B-type natriuretic peptide in cardiovascular disease. Lancet 2003; 362(9380): 316-22.

45. Wright GA, Struthers AD. Natriuretic peptides as a prognostic marker and therapeutic target in heart failure. Heart 2006; 92(2): 149-51.

46. Adelmann D, Kronish K, Ramsay MA. Anesthesia for Liver Transplantation. Anesthesiol Clin. 2017; 35(3): 491-508. 